\title{
Decomposition of Generalized Asymmetry Model for Square Contingency Tables
}

\author{
Shuji Ando1, Hiroyuki Kurakami² \\ ${ }^{1}$ Data Management \& Biostatistics Group Re-Examination Department, Novartis Pharma K.K., Tokyo, Japan \\ ${ }^{2}$ Department of Information Sciences, Faculty of Science and Technology, \\ Tokyo University of Science, Chiba, Japan \\ Email: shuji.ando@novartis.com,kurakami@rs.tus.ac.jp
}

Received 18 April 2016; accepted 11 June 2016; published 14 June 2016

Copyright (C) 2016 by authors and Scientific Research Publishing Inc.

This work is licensed under the Creative Commons Attribution International License (CC BY).

http://creativecommons.org/licenses/by/4.0/

(c) (i) Open Access

\section{Abstract}

For the analysis of square contingency tables with same row and column ordinal classifications, the present paper gives the decomposition of the generalized linear diagonals-parameter symmetry model using the diagonals-parameter symmetry model. Moreover, it gives the decomposition of the symmetry model using above the proposed decomposition.

\section{Keywords}

Diagonals-Parameter Symmetry, Linear Diagonals-Parameter Symmetry, Orthogonality, Symmetry

\section{Introduction}

Consider an $R \times R$ square contingency table with the same row and column classifications. Let $p_{i j}$ denote the probability that an observation will fall in the $i$ th row and $j$ th column of the table $(i=1, \cdots, R ; j=1, \cdots, R)$. For square tables with ordered categories, Goodman [1] proposed the diagonals-parameter symmetry (DPS) model, defined by

$$
p_{i j}= \begin{cases}\delta_{j-i} \psi_{i j} & (i<j), \\ \psi_{i j} & (i \geq j),\end{cases}
$$

where $\psi_{i j}=\psi_{j i}$. Note that the DPS models with $\left\{\delta_{j-i}=1\right\},\left\{\delta_{j-i}=\delta^{j-i}\right\}$, and $\left\{\delta_{j-i}=\delta^{R-(j-i)}\right\}$ are identical to the symmetry (S) (Bowker [2]), linear diagonals-parameter symmetry (LDPS) (Agresti [3]), and another LDPS (ALDPS) (Tomizawa [4]) models, respectively. 
Yamamoto and Tomizawa [5] proposed the generalization of LDPS model. We will denote $\mathbb{Z}$ as the set of integers. For a fixed $K \in \mathbb{Z}$, the generalized LDPS (LDPS $(K)$ ) model is defined by

$$
p_{i j}= \begin{cases}\delta^{K+(j-i)} \psi_{i j} & (i<j), \\ \psi_{i j} & (i \geq j),\end{cases}
$$

where $\psi_{i j}=\psi_{j i}$. Note that the $\operatorname{LDPS}(K)$ model with $\delta=1$ is identical to the S model. Especially the LDPS(0) and LDPS(-R) models are equivalent to the LDPS and ALDPS models, respectively.

Tomizawa [6] gave the decomposition of the LDPS model using the DPS model, and showed that a test statistic for the LDPS model was equal to the sum of those for decomposed models.

For the analysis of square contingency tables with ordered categories, the purposes of this paper are (1) to give the decomposition of the LDPS $(K)$ model using the DPS model, (2) to show that for the test statistic for the LDPS $(K)$ model is equal to the sum of those for decomposed models, and (3) to give the decomposition of the $S$ model using above the decomposition of the $\operatorname{LDPS}(K)$ model.

\section{Decomposition of the Generalized Asymmetry Model}

Tomizawa [6] proposed the linear diagonals-parameter marginal symmetry (LDPMS) model, defined by

$$
p_{(i)}^{+}=\Delta^{i} p_{(i)}^{-} \quad(i=1, \cdots, R-1),
$$

where

$$
p_{(i)}^{+}=\sum_{s=1}^{R-i} p_{s, s+i}, \quad p_{(i)}^{-}=\sum_{s=1}^{R-i} p_{s+i, s} .
$$

Let $X$ and $Y$ denote the row and column variables, respectively. The LDPMS model indicates that $\operatorname{Pr}(Y-X=i)$ is $\Delta^{i}$ times higher than $\operatorname{Pr}(X-Y=i)$ for all $i=1, \cdots, R-1$. Under LDPMS model, if $\Delta>1$ then $\operatorname{Pr}(X<Y)>\operatorname{Pr}(X>Y)$, and if $\Delta<1$ then $\operatorname{Pr}(X<Y)<\operatorname{Pr}(X>Y)$.

Also, Tomizawa [6] gave the decomposition of the LDPS model using the DPS and LDPMS models, and showed that a test statistic for the LDPS model is equal to the sum of those for the DPS and LDPMS models.

To consider the decomposition of the $\operatorname{LDPS}(K)$ model, we shall introduce a new model. For a fixed $K \in \mathbb{Z}$, the generalized LDPMS (LDPMS $(K))$ model is defined by

$$
p_{(i)}^{+}=\Delta^{K+i} p_{(i)}^{-} \quad(i=1, \cdots, R-1) .
$$

Especially the LDPMS(0) model is equivalent to the LDPMS model.

We will denote $\mathbb{Z}_{1}$ as the set of integers of $-R+1$ or less, $\mathbb{Z}_{2}$ as the set of integers from $-R+2$ to -2 , and $\mathbb{Z}_{3}$ as the set of integers of -1 or greater. Under the LDPMS $(K)$ model with a fixed $K \in \mathbb{Z}_{1}$, if $\Delta>1$ then $\operatorname{Pr}(X<Y)<\operatorname{Pr}(X>Y)$. Also, under the LDPMS $(K)$ model with a fixed $K \in \mathbb{Z}_{2}$, if $\Delta>1$, there exists a certain $t$ such that $\operatorname{Pr}(Y-X=i) \leq \operatorname{Pr}(X-Y=i)$ for $i=1,2, \cdots, t$ and $\operatorname{Pr}(Y-X=i)>\operatorname{Pr}(X-Y=i)$ for $i=t+1, t+2, \cdots, R-1$. Moreover, under the LDPMS $(K)$ model with a fixed $K \in \mathbb{Z}_{3}$, if $\Delta>1$ then $\operatorname{Pr}(X<Y)>\operatorname{Pr}(X>Y)$.

We obtain the following theorem.

Theorem 1. For a fixed $K \in \mathbb{Z}$, the LDPS(K) model holds if and only if both the DPS and LDPMS(K) models hold.

Proof. If the LDPS $(K)$ model holds, then the DPS and LDPMS $(K)$ models hold. Assuming that both the DPS and LDPMS $(K)$ models hold, then we shall show that the LDPS $(K)$ model holds.

From the LDPMS $(K)$ model holds, we obtain

$$
\sum_{s=1}^{R-i} p_{s, s+i}=\Delta^{K+i} \sum_{s=1}^{R-i} p_{s+i, s} \quad(i=1, \cdots, R-1) .
$$

Also, from the DPS model holds, we see

$$
\sum_{s=1}^{R-i} p_{s, s+i}=\delta_{i} \sum_{s=1}^{R-i} p_{s+i, s} \quad(i=1, \cdots, R-1) .
$$


Therefore, we obtain $\Delta^{K+i}=\delta_{i}$ for all $i=1, \cdots, R-1$. Namely, the LDPS $(K)$ model holds. The proof is completed.

\section{Orthogonality of Test Statistic and Model Selection}

Assume that a multinomial distribution applies to the $R \times R$ table. Let $n_{i j}$ denote the observed frequency in the $i$ th row and $j$ th column of the $R \times R$ square table $(i=1, \cdots, R ; j=1, \cdots, R)$, with $n=\sum \sum n_{i j}$. The maximum likelihood estimates (MLEs) of expected frequencies under the model could be obtained by using, e.g., the Newton-Raphson method in the log-likelihood equation.

Each model can be tested for goodness-of-fit by, e.g., the likelihood ratio chi-square statistic (denoted by $G^{2}$ ) with the corresponding degrees of freedom (df). The test statistic $G^{2}$ of model $M$ is given by

$$
G^{2}(M)=2 \sum_{i=1}^{R} \sum_{j=1}^{R} n_{i j} \log \left(\frac{n_{i j}}{\hat{m}_{i j}}\right),
$$

where $\hat{m}_{i j}$ is the MLE of expected frequency $m_{i j}$ under model $M$. The number of df for LDPMS $(K)$ model is $R-2$, which is equal to that for LDPMS model.

A quick method for choosing the best-fitting model among different models is to use Akaike's [7] information criterion (AIC), which is defined as

$$
\text { AIC }=-2(\text { maximum log likelihood })+2(\text { number of parameters }),
$$

for each model. For more details of AIC, see Konishi and Kitagawa [8]. This criterion gives the best-fitting model as the one with minimum AIC. Since only the difference between AICs is required when two models are compared, it is possible to ignore a common constant of AIC and we may use a modified AIC defined as

$$
\mathrm{AIC}^{+}=G^{2}-2(\text { number of df }) \text {. }
$$

Thus, for the data, the model with the minimum $\mathrm{AIC}^{+}$(i.e., the minimum AIC) is the best-fitting model.

For the analysis of contingency tables, Read [9] discussed the orthogonality, which is equivalent to the asymptotic separability in Aitchison [10] and the independence in Darroch and Silvey [11] of test statistic for goodness-of-fit of two models.

On the orthogonality of test statistic for models in Theorem 1, we obtain the following theorem.

Theorem 2. For a fixed $K \in \mathbb{Z}$, the following equation holds:

$$
G^{2}(\operatorname{LDPS}(K))=G^{2}(\mathrm{DPS})+G^{2}(\operatorname{LDPMS}(K)) \text {. }
$$

The number of $d f$ for the LDPS(K) model equals the sum of number of $d f$ for the DPS and LDPMS(K) models.

Proof. First, we consider that the MLEs of expected frequencies $\left\{m_{i j}\right\}$ under the LDPS $(K)$ model are given by

$$
\hat{m}_{i j}= \begin{cases}\frac{\hat{\delta}^{K+j-i}}{1+\hat{\delta}^{K+j-i}} \cdot\left(n_{i j}+n_{j i}\right) & \text { for } i<j \\ \frac{1}{1+\hat{\delta}^{K+i-j}} \cdot\left(n_{i j}+n_{j i}\right) & \text { for } i>j \\ n_{i j} & \text { for } i=j,\end{cases}
$$

where $\hat{\delta}$ is the solution of the following equation

$$
\sum_{l=1}^{R-1}(K+l)\left(n_{(l)}^{+}-\delta^{K+l} n_{(l)}^{-}\right) /\left(1+\delta^{K+l}\right)=0,
$$

with

$$
n_{(l)}^{+}=\sum_{s=1}^{R-l} p_{s, s+l}, \quad n_{(l)}^{-}=\sum_{s=1}^{R-l} p_{s+l, s} .
$$

We can solve (3.1) for $\delta$ by using the Newton-Raphson method. 
Second, we consider that the MLEs of expected frequencies $\left\{m_{i j}\right\}$ under the DPS model are given by

$$
\hat{m}_{i j}= \begin{cases}\frac{\hat{\delta}_{j-i}}{1+\hat{\delta}_{j-i}} \cdot\left(n_{i j}+n_{j i}\right) & \text { for } i<j \\ \frac{1}{1+\hat{\delta}_{i-j}} \cdot\left(n_{i j}+n_{j i}\right) & \text { for } i>j \\ n_{i j} & \text { for } i=j,\end{cases}
$$

where $\hat{\delta}_{j-i}=n_{(j-i)}^{+} / n_{(j-i)}^{-}$.

Last, we consider that the MLEs of expected frequencies $\left\{m_{i j}\right\}$ under the LDPMS $(K)$ model are given by

$$
\hat{m}_{i j}= \begin{cases}\frac{\hat{\Delta}^{K+j-i}}{1+\hat{\Delta}^{K+j-i}} \cdot \frac{n_{(j-i)}^{+}+n_{(j-i)}^{-}}{n_{(j-i)}^{+}} \cdot n_{i j} & \text { for } i<j \\ \frac{1}{1+\hat{\Delta}^{K+i-j}} \cdot \frac{n_{(i-j)}^{+}+n_{(i-j)}^{-}}{n_{(i-j)}^{-}} \cdot n_{i j} & \text { for } i>j \\ n_{i j} & \text { for } i=j,\end{cases}
$$

where $\hat{\Delta}$ is the solution of the Equation (3.1) with $\delta$ replaced by $\Delta$. Thus, we see that $\left(n_{i j} / \hat{m}_{i j}\right)$ under the $\operatorname{LDPS}(K)$ model is equal to the product of $\left(n_{i j} / \hat{m}_{i j}\right)$ under the DPS model and that under the LDPMS $(K)$ model. Therefore, the test statistic for goodness-of-fit for $\operatorname{LDPS}(K)$ model is equal to the sum of those for two models. The proof is completed.

\section{Decomposition of the Symmetry Model}

For square contingency tables with ordered categories, Kurakami, Yamamoto and Tomizawa [12] considered two models. One is the generalized exponential symmetry (GES) model defined by

$$
p_{i j}= \begin{cases}\gamma^{w_{i j}} \psi_{i j} & (i<j), \\ \psi_{i j} & (i \geq j),\end{cases}
$$

where $\psi_{i j}=\psi_{j i}$ and $\left\{w_{i j}\right\}$ are the specified non-negative values. The other is the generalized weighted global symmetry (GWGS) model defined by

$$
\sum_{i<j} w_{i j} p_{i j}=\sum_{i<j} w_{i j} p_{j i}
$$

For a fixed $K \in \mathbb{Z}_{3}$, the GES model with non-negative values $\left\{w_{i j}=K+j-i\right\}$ is identical to the LDPS(K) model. For a fixed $K \in \mathbb{Z}_{1}$, because $\left\{w_{i j}=-(K+j-i)\right\}$ are non-negative values, the LDPS $(K)$ model is included in the GES model. Note that for a fixed $K \in \mathbb{Z}_{2}$, the LDPS $(K)$ model is not included in the the GES model, because $\left\{w_{i j}=K+j-i\right\}$ have both positive and negative values. For a fixed $K \in \mathbb{Z}_{1} \cup \mathbb{Z}_{3}$, we shall refer to the GWGS model with $\left\{w_{i j}=|K+j-i|\right\}$ as the WGS $(K)$ model.

Kurakami et al. [12] also gave the decomposition of the S model using the GES and GWGS models, and showed that a test statistic for the $\mathrm{S}$ model is approximately equivalent to the sum of those for the GES and GWGS models.

We will denote $\mathbb{Z}_{4}$ as the set of non-negative integers. Yamamoto, Ohama and Tomizawa [13] gave the following theorems.

Theorem 3. For a fixed $K \in \mathbb{Z}_{4}$, the $S$ model holds if and only if both the LDPS(K) and WGS(K) models hold.

Theorem 4. For a fixed $K \in \mathbb{Z}_{4}$, the following asymptotic equivalence holds:

$$
G^{2}(S) \simeq G^{2}(\operatorname{LDPS}(K))+G^{2}(\operatorname{WGS}(K)) .
$$

The number of $d f$ for the $S$ model equals the sum of the number of $d f$ for the LDPS(K) and WGS(K) models. 
From the theorems given by Kurakami et al. [12], we obtain the following theorems as extensions of Theorems 3 and 4 (because the $\mathbb{Z}_{1} \cup \mathbb{Z}_{3}$ includes $\mathbb{Z}_{4}$ ).

Theorem 5. For a fixed $K \in \mathbb{Z}_{1} \cup \mathbb{Z}_{3}$, the $S$ model holds if and only if both the LDPS(K) and WGS(K) models hold.

Theorem 6. For a fixed $K \in \mathbb{Z}_{1} \cup \mathbb{Z}_{3}$, the following asymptotic equivalence holds:

$$
G^{2}(S) \simeq G^{2}(\operatorname{LDPS}(K))+G^{2}(\operatorname{WGS}(K)) \text {. }
$$

The number of $d f$ for the $S$ model equals the sum of the number of $d f$ for the LDPS(K) and WGS(K) models.

From Theorems 1 to 6, we obtain the following corollaries.

Corollary 1. For a fixed $K \in \mathbb{Z}_{1} \cup \mathbb{Z}_{3}$, the $S$ model holds if and only if all the DPS, LDPMS(K) and WGS(K) models hold.

Corollary 2. For a fixed $K \in \mathbb{Z}_{1} \cup \mathbb{Z}_{3}$, the following asymptotic equivalence holds:

$$
G^{2}(S) \simeq G^{2}(\mathrm{DPS})+G^{2}(\operatorname{LDPMS}(K))+G^{2}(\operatorname{WGS}(K)) .
$$

The number of $d f$ for the $S$ model equals the sum of the number of $d f$ for the DPS, LDPMS(K) and WGS(K) models.

\section{An Example}

Consider the data in Table 1, taken directly from Bishop, Fienberg and Holland ([14], p. 100). From Table 2, all LDPS $(K)$ models, the $S$ model and DPS model give poor fits to these data. However, all LDPMS $(K)$ models fit these data well.

The LDPMS(2) model is the best-fitting model among the other LDPMS $(K)$ models because it has a minimum $\mathrm{AIC}^{+}$value. Under the LDPMS(2) model, the MLE of $\Delta$ is $\hat{\Delta}=1.04$. Thus, we see that the status category for a father tends to be less than that for his son.

Theorem 1 would be useful for seeing the reason for its poor fit when the LDPS $(K)$ model fits the data poorly. Thus, for the data in Table 1, the poor fit of the LDPS $(K)$ model is caused by the poor fit of the DPS model rather than the LDPMS $(K)$ model. Also, Theorem 5 would be useful for seeing the reason for its poor fit when the S model fits the data poorly. From Table 2, WGS $(K)$ models (except the WGS(-1) model) give poor fits to these data. Thus, when $K$ is not equal to -1 , we cannot see that the poor fit of the $S$ model is caused by the poor fit of either LDPS $(K)$ and WGS $(K)$ models (although, we can see that the poor fit of the S model is caused by the poor fit of both LDPS $(K)$ and WGS(K) models). However, using Corollary 1, we can see that the poor fit of

Table 1. Occupational status for Danish father-son pairs; from Bishop et al. ([14], p. 100) (The parenthesized value is MLEs

\begin{tabular}{|c|c|c|c|c|c|c|}
\hline \multirow{2}{*}{ Father's status } & \multicolumn{5}{|c|}{ Son's status } & \multirow{2}{*}{ Total } \\
\hline & (1) & (2) & (3) & (4) & (5) & \\
\hline \multirow[t]{2}{*}{ (1) } & 18 & 17 & 16 & 4 & 2 & 57 \\
\hline & (18.00) & (17.06) & (15.80) & (3.61) & $(4.48)$ & \\
\hline \multirow[t]{2}{*}{ (2) } & 24 & 105 & 109 & 59 & 21 & 318 \\
\hline & (23.90) & (105.00) & $(109.41)$ & (58.25) & (18.93) & \\
\hline \multirow[t]{2}{*}{ (3) } & 23 & 84 & 289 & 217 & 95 & 708 \\
\hline & (23.35) & (83.65) & $(289.00)$ & (217.82) & (93.79) & \\
\hline \multirow[t]{2}{*}{ (4) } & 8 & 49 & 175 & 348 & 198 & 778 \\
\hline & $(9.23)$ & (49.75) & $(174.26)$ & (348.00) & (198.75) & \\
\hline \multirow[t]{2}{*}{ (5) } & 6 & 8 & 69 & 201 & 246 & 530 \\
\hline & $(3.52)$ & $(9.23)$ & (70.06) & (200.15) & $(246.00)$ & \\
\hline Total & 79 & 263 & 658 & 829 & 562 & 2391 \\
\hline
\end{tabular}
of expected frequencies under the LDPMS (2) model).

Note: Status (1) is high professionals, (2) White-collar employees of higher education, (3) White-collar employees of less high education, (4) Upper working class, and (5) Unskilled workers. 
Table 2. Likelihood ratio chi-square values $G^{2}$ and $\mathrm{AIC}^{+}$for models applied to the data in Table 1.

\begin{tabular}{|c|c|c|c|}
\hline Applied models & Df & $G^{2}$ & $\mathrm{AIC}^{+}$ \\
\hline S & 10 & $24.80^{*}$ & 4.80 \\
\hline DPS & 6 & $14.84^{*}$ & 2.84 \\
\hline $\operatorname{LDPS}(-5)$ & 9 & $19.21^{*}$ & 1.21 \\
\hline $\operatorname{LDPS}(-4)$ & 9 & $19.41^{*}$ & 1.41 \\
\hline $\operatorname{LDPS}(-3)$ & 9 & $19.91^{*}$ & 1.91 \\
\hline $\operatorname{LDPS}(-2)$ & 9 & $21.94^{*}$ & 3.94 \\
\hline $\operatorname{LDPS}(-1)$ & 9 & $22.56^{*}$ & 4.56 \\
\hline LDPS(0) & 9 & $19.05^{*}$ & 1.05 \\
\hline LDPS(1) & 9 & $18.72^{*}$ & 0.72 \\
\hline LDPS(2) & 9 & $18.68^{*}$ & 0.68 \\
\hline LDPS(3) & 9 & $18.68^{*}$ & 0.68 \\
\hline LDPS(4) & 9 & $18.69^{*}$ & 0.69 \\
\hline LDPS(5) & 9 & $18.71^{*}$ & 0.71 \\
\hline LDPMS(-5) & 3 & 4.37 & -1.63 \\
\hline LDPMS(-4) & 3 & 4.57 & -1.43 \\
\hline LDPMS(-3) & 3 & 5.07 & -0.93 \\
\hline LDPMS(-2) & 3 & 7.11 & 1.11 \\
\hline LDPMS(-1) & 3 & 7.72 & 1.72 \\
\hline LDPMS(0) & 3 & 4.22 & -1.78 \\
\hline LDPMS(1) & 3 & 3.89 & -2.11 \\
\hline LDPMS(2) & 3 & 3.84 & -2.16 \\
\hline LDPMS(3) & 3 & 3.85 & -2.15 \\
\hline LDPMS(4) & 3 & 3.86 & -2.14 \\
\hline LDPMS(5) & 3 & 3.87 & -2.13 \\
\hline WGS(-5) & 1 & $5.59^{*}$ & 3.59 \\
\hline WGS(-4) & 1 & $5.39^{*}$ & 3.39 \\
\hline WGS $(-1)$ & 1 & 2.22 & 0.22 \\
\hline WGS(0) & 1 & $5.73^{*}$ & 3.73 \\
\hline WGS(1) & 1 & $6.07^{*}$ & 4.07 \\
\hline WGS(2) & 1 & $6.12^{*}$ & 4.12 \\
\hline WGS(3) & 1 & $6.12^{*}$ & 4.12 \\
\hline WGS(4) & 1 & $6.11^{*}$ & 4.11 \\
\hline WGS(5) & 1 & $6.10^{*}$ & 4.10 \\
\hline
\end{tabular}

*Means significant at the 0.05 level.

the S model is caused by the poor fit of DPS and WGS $(K)$ models rather than the LDPMS $(K)$ model.

\section{Concluding Remarks}

We have given the decomposition of the LDPS(K) model using the DPS model (namely, Theorem 1 ). Also, we 
have shown that the test statistic for the $\operatorname{LDPS}(K)$ is equal to the sum of those for the decomposed models (namely, Theorem 2). Moreover, we have given the decomposition of the S model using Theorem 1 (namely, Corollary 1 ), and shown that the test statistic for the $S$ model is approximately equivalent to the sum of those for the decomposed models (namely, Corollary 2). Although details will be omitted, Yamamoto, Ohama and Tomizawa [15] gave the another decomposition of the the LDPS(K) model for a fixed $K \in \mathbb{Z}_{4}$. However, it does not hold the orthogonality of test statistic for models. Thus, Theorem 1 may be useful for analyzing the data than the decomposition by Yamamoto et al. [15]. Because Theorem 1 shows the decomposition of LDPS(K) for a fixed $K \in \mathbb{Z}$ (because $\mathbb{Z}$ includes $\mathbb{Z}_{4}$ ), and also holds the orthogonality of test statistic for models.

\section{Acknowledgements}

We thank the reviewer for the helpful comments. Also, we thank Professor S. Tomizawa and Dr. K. Tahata of Tokyo University of Science for their useful suggestions.

\section{References}

[1] Goodman, L.A. (1979) Multiplicative Models for Square Contingency Tables with Ordered Categories. Biometrika, 66, 413-418. http://dx.doi.org/10.1093/biomet/66.3.413

[2] Bowker, A.H. (1948) A Test for Symmetry in Contingency Tables. Journal of the American Statistical Association, 43, 572-574. http://dx.doi.org/10.1080/01621459.1948.10483284

[3] Agresti, A. (1983) A Simple Diagonals-Parameter Symmetry and Quasi-Symmetry Model. Statistics and Probability Letters, 1, 313-316. http://dx.doi.org/10.1016/0167-7152(83)90051-2

[4] Tomizawa, S. (1990) Another Linear Diagonals-Parameter Symmetry Model for Square Contingency Tables with Ordered Categories. South African Statistical Journal: Journal of the South African Statistical Association, 24, 117-125.

[5] Yamamoto, K. and Tomizawa, S. (2012) Statistical Analysis of Case-Control Data of Endometrial Cancer Based on New Asymmetry Models. Journal of Biometrics and Biostatistics, 3, 1-4. http://dx.doi.org/10.4172/2155-6180.1000147

[6] Tomizawa, S. (1990) A Decomposition of Linear Diagonals-Parameter Symmetry Model for Square Contingency Tables with Ordered Categories. Biometrical Journal, 32, 761-766. http://dx.doi.org/10.1002/bimj.4710320619

[7] Akaike, H. (1974) A New Look at the Statistical Model Identification. IEEE Transactions on Automatic Control, AC19, 716-723. http://dx.doi.org/10.1109/TAC.1974.1100705

[8] Konishi, S. and Kitagawa, G. (2008) Information Criteria and Statistical Modeling. Springer, New York. http://dx.doi.org/10.1007/978-0-387-71887-3

[9] Read, C.B. (1977) Partitioning Chi-Square in Contingency Table: A Teaching Approach. Communications in Statistics-Theory and Methods, 6, 553-562. http://dx.doi.org/10.1080/03610927708827513

[10] Aitchson, J. (1962) Large-Sample Restricted Parametric Tests. Journal of the Royal Statistical Society, Series B, 24, 234-250.

[11] Darroch, J.N. and Silvey, S.D. (1963) On Testing More than One Hypothesis. Annals of Mathematical Statistics, 34, 555-567. http://dx.doi.org/10.1214/aoms/1177704168

[12] Kurakami, H., Yamamoto, K. and Tomizawa, S. (2011) Generalized Exponential Symmetry Model and Orthogonal Decomposition of Symmetry for Square Tables. The Open Statistics and Probability Journal, 3, 1-6. http://dx.doi.org/10.2174/1876527001103010001

[13] Yamamoto, K., Ohama, M. and Tomizawa, S. (2013) Decompositions of Symmetry Using Generalized Linear Diagonals-Parameter Symmetry Model and Orthogonality of Test Statistic for Square Contingency Tables. Open Journal of Statistics, 3, 9-13. http://dx.doi.org/10.4236/ojs.2013.36A002

[14] Bishop, Y.M.M., Fienberg, S.E. and Holland, P.W. (1975) Discrete Multivariate Analysis: Theory and Practice. The MIT Press, Cambridge.

[15] Yamamoto, K., Ohama, M. and Tomizawa, S. (2015) Vision Data Analysis Based on New Statistical Models and Decompositions. Annals of Biometrics and Biostatistics, 2, 1-7. 